\title{
Deuterium Depleted Water: Non-Nuclear Applications in Medicines
}

\author{
Archana Dhyani, Vijay Kumar, Nardev Singh, R.C Joshi
}

\begin{abstract}
Normal water contains $150 \mathrm{ppm}$ of deuterium; however DDW contains less amount of deuterium. The literature review indicated that this unique property of DDW may be used as hepato protective, antioxidant, in the treatment of hepatorenal toxicity, anti-inflammatory, colorectal cancer, hypertension, diabetes, metabolic disorders and obesity. This article suggests that there is tremendous scope of using deuterium depleted water in the treatment of various diseases.
\end{abstract}

Keywords: Deuterium depleted water, Therapeutic applications, Diseases, Treatment.

\section{INTRODUCTION}

Hydrogen has three isotopes hydrogen, deuterium and tritium. Deuterium atom having one neutron extra than hydrogen atom. The concentration of deuterium, which is an isotope of hydrogen in normal water is $150 \mathrm{ppm}$. The natural abundance of deuterium in DDW is less as compared to natural water. It is reported that DDW of $25 \mathrm{ppm}$ to 120 ppm has numerous positive health application like anticancer/tumour, fight against side effects of chemotherapy and radiotherapy, elimination of DNA errors and in treatment of diabetes, heart diseases, thalassemia, nonspecific immune defence of the body, anti-ageing and radio-protective effects. Heavy Water Board Plants produce Heavy Water and DDW in different concentration (between $30 \mathrm{ppm}$ and 125ppm). After recognizing the increasing potential of DDW, Heavy Water Board, Mumbai promotes the utilization of it in collaboration with Medical Fraternity, Indian Companies, Research Institutes/Academia. ${ }^{[1]}$

\section{METHODS FOR DEUTERIUM DEPLETED WATER}

At present, the light water was obtained from the method of distillation using large columns ${ }^{[2]}$. The process consists vacuum distillation of natural water on column capable of highly performing ordered packing. The system allows obtaining DDW at concentration within the range of 20-120 ppm. $^{[3]}$

Revised Manuscript Received on April 25, 2019.

*Corresponding Author

*Archana Dhyani, School of Pharmacy, Graphic Era Hill University, Clement Town, Dehradun, Uttarakhand, India. E-mail: archana.dhyani89@gmail.com

Vijay Kumar, Department of Allied Sciences, Graphic Era Hill University, Clement Town, Dehradun, Uttarakhand, India

Nardev Singh, School of Pharmacy, Graphic Era Hill University, Clement Town, Dehradun, Uttarakhand, India.

R.C Joshi, Department of Chemistry, Graphic Era University, Clement Town, Dehradun, Uttarakhand, India.

\section{THERAPEUTIC APPLICATIONS}

Deuterium Depleted Water finds its application in treatment of sepsis induced by cecal ligation and puncture, which occurs due to reactive species. Both, M. longifolia oil and DDW show antioxidation as well as hepatoprotection activities. The study was conducted by Azadeh Rasoolia et al (2019). The study concludes that both DDW and M. longifolia reduce the oxidation reaction as well as inflammation. ${ }^{[4]}$ DDW has potential to antagonize the manganese (Mn)-induced toxicity which leads to decrease in survival period. For this study, Caenorhabditis elegans was used as replica. DDW reduces the DAF-16 levels, the factor responsible for life span. ${ }^{[5]}$ This study reported by Daiana Silva Ávila and co-workers (2012). The ability of DDW to treat hepatorenal toxicity was determined by Stepan Sergeevichto and his team (2018) on healthy Wistar rats. The amount of creatinine, bilirubin, aspartate aminotransferase and alanine aminotransferase were deduced with DDW treatment. ${ }^{[6]}$

The in-vivo and in vitro inhibition effect of DDW on lung cancer was studied by Feng-song Cong and co-workers (2010). The study involved the treatment of lung cancer cell line A549 and lung fibroblasts HLF-1 with different concentration of DDW. ${ }^{[7]}$ DDW reduces the enlargement of cancer cell lines and suppress oncogenes. The study was done by Zoltán Gyöngyi et al (2013) on 129 patients who had tiny cell and large cell lung cancers. The patients consumed DDW in additional to chemotherapy and radiotherapy. It was observed that the survival period was increased in patients consuming DDW. ${ }^{[8]}$

Sergiu Chira and its co-workers (2018) studied the effect of DDW on DLD-1 colorectal cancer models. To determine this, different functional assays were carry out, including apoptosis, autophagy mitochondrial activity and senescence assays. ${ }^{[9]}$ The affect of DDW $55 \pm 5$ ppm on 12 week old Wistar-Kyoto rats (WKY) and spontaneously hypertensive rats (SHR) were studied by R. Rehakova and its co-workers (2016). The biochemical parameters and Blood pressure (BP) were monitored and it was found that DDW is able to reduce cholesterol and triglyceride levels. [10]

The use of DDW on working memory and locomotor activity was done by Cristian Mladin et al (2014). However, the results show that there is no effect of DDW on locomotor activity and working memory of wistar rat. ${ }^{[11]}$ DDW finds an interesting application in treatment of metabolic diseases. Ignat Ignatov et al (2014) performed the clinical trials of DDW (60-100 ppm), proved that DDW can be given in treatment of metabolic disorders such as rise in $\mathrm{BP}$, obesity, impaired glucose metabolism and diabetes.

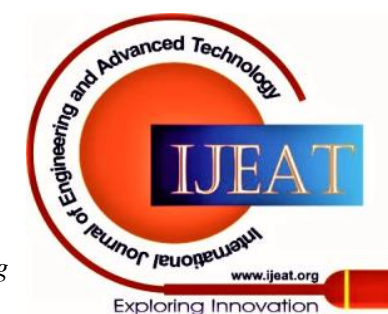


It was also concluded the DDW can be used to treat nephrolithiasis and in the gastrointestinal tract disrders. ${ }^{[12]}$ The use of DDW on tumor growth was done by László G. Boros et al (2016) who studied that DDW suppresses the progression of tumor in mice, dogs, cats and human. A model was proposed in which electron transport chain (ETC) decreases oxygen which finally affect gluconeogenesis and oxidation of fats as well. It can help in cancer prevention and treatment suitable diets. ${ }^{[13]}$ The relationship between the use of DDW and normal water in model of mouse of chronic mild stress was done by Tatyana Strekalova and team (2014) . It was found the depression will be reduced in mice provided with DDW in comparison to normal water ${ }^{[14]}$. The use of DDW on nasopharyngeal carcinoma cell lines was done by Hongqiang Wanga and co-workers (2013) in which the nasopharyngeal carcinoma cells and preosteoblast cells were grown in media having diverse concentration of deuterium (50-150 ppm). This property of inhibiting the cell lines were done by plate colony formation, Transwell assays, western blot and immunofluorescence. It was effective in inhibition and suppression of cancer cells.(15)

Table 1: DDW uses in different diseases

\begin{tabular}{|c|c|c|}
\hline & Type of Cells & Effects \\
\hline 1 & Lung cancer cell & $\begin{array}{c}\text { DDW decreases the cancer } \\
\text { cell lines and suppress } \\
\text { oncogenes }\end{array}$ \\
\hline 2 & $\begin{array}{c}\text { DLD-1colorectal } \\
\text { cancer }\end{array}$ & $\begin{array}{c}\text { DDW can be modulator of } \\
\text { adjuvant therapy }\end{array}$ \\
\hline 3 & $\begin{array}{c}\text { Hypertension } \\
\text { DDW is able to reduce } \\
\text { cholesterol and triglyceride } \\
\text { levels and helps in reduction } \\
\text { of hypertension }\end{array}$ \\
\hline 5 & $\begin{array}{c}\text { Metabolic } \\
\text { Disorders } \\
\text { tumor }\end{array}$ & $\begin{array}{c}\text { DDW can be given in } \\
\text { treatment of metabolic } \\
\text { disorders }\end{array}$ \\
\hline 7 & $\begin{array}{c}\text { Nasopharyngeal } \\
\text { carcinoma cell } \\
\text { lines }\end{array}$ & $\begin{array}{c}\text { DDW helps in cancer } \\
\text { treatment. }\end{array}$ \\
\hline & DDW inhibits the cell lines \\
\hline
\end{tabular}

\section{CONCLUSION}

This review discussed the therapeutic effects of DDW in healing of various diseases. The different researchers use DDW in the management of different ailments. From medicinal point of view, DDW can use as solvent for treating various disorders like cancer, hyperglycemia, hepatoprotective, antiinflammmatory, and antioxidant. Thus, it can be concluded that deuterium depleted water (DDW) may be medicinally used in future.

\section{REFERENCES}

1. http://www.hwb.gov.in.

2. Patent № 2295493 Russian Federation, IPC C01D5/00, B01D59/00, B01D59/02, B01D3/14. The process method and the plant for light water production. / S.P. Solov'yev, - priority 28.05.2004

3. 3.Barishev M.G.,Dzhimak S.S., Frolov V.U , Bolotin S.N.,Dolgov M.A.,Technologies For Obtaining Deuterium Depleted Water, International Journal of Engineering Research and Applications,2013, 3(1): 523-526.

4. Rasoolia Azadeh, Fatemib Faezeh, Hajihosseinia Reza, Vaziria Atoosa, Akbarzadehc Kambiz, Malayerid Mohammad Reza Mohammadi, Dinie Salome and Foroutanrad Maria, Synergistic Effects Of Deuterium Depleted Water And Mentha Longifolia L. Essential Oils On Sepsis-Induced Liver Injuries Through Regulation Of Cyclooxygenase-2, Pharmaceutical Biology 2019, 57(1):125-132.

5. Ávilaa Daiana Silva , Somlyaib Gábor, Somlyai Ildikó and Aschner Michael, Anti-aging effects of deuterium depletion on Mn-induced toxicity in a C. elegans model, Toxicology Letters 2012, 211(3): 319324.

6. Sergeevich Dzhimak Stepan, Basov Alexandr Alexandrovich,Elkina Anna Anatolyevna,Fedulova Liliya Viacheslavovna, Kotenkova Elena Alexandrovna, Vasilevskaya Ekaterina Romanovna, Lyasota Oxana Mikhailovna, and Baryshev Mikhail Gennadievich, Influence of Deuterium-Depleted Water on Hepatorenal Toxicity, Jundishapur J Nat Pharm Prod. 2018 ,vol.13,no.2:1-5

7. Feng-song Cong, Ya-ru Zhang, Hong-cai Sheng, Zong-huaAo, Su-yi Zhang and Ju-yong Wang, Deuterium-depleted water inhibits human lung carcinoma cell growth by apoptosis, Experimental And Therapeutic Medicine 2010,vol. 1: 277-283.

8. Zoltan Gyongyi, Ferenc Budan, Istvan Szabo, Istvan Ember, and Istvan Kiss, Deuterium Depleted Water Effects on Survival of Lung Cancer Patients and Expression of Kras, Bcl2,and Myc Genes in Mouse Lung, Nutrition and Cancer, 2013,vol.65,no.2, 240-246

9. Sergiu Chira, Lajos Raduly, Cornelia Braicu, Ancuta Jurj, Roxana Cojocneanu-Petric, Laura Pop1, Valentina Pileczki1, Calin Ionescu And Ioana Berindan-Neagoe, Premature Senescence Activation In DLD-1 Colorectal Cancer Cells Through Adjuvant Therapy To Induce A Mirna Profile Modulating Cellular Death, Experimental And Therapeutic Medicine,2018,vol 16: 1241-1249.

10. R. Rehakova, J. Klimentova, M. Cebova1, A. Barta, Z. Matuskova,P Labas, O. Pechanova, Effect of Deuterium-Depleted Water on Selected Cardiometabolic Parameters in Fructose-Treated Rats, Physiological Research 2016,vol 65,no. 3: S401-S407.

11. Cristian Mladin, Alin Ciobica, Radu Lefter, Alexandru Popescu And Walther Bild, Deuterium Depletion Induces Anxiolytic-Like Effects In Rats, Arch. Biol. Sci., Belgrade, 2014, vol.66,no.2, 947-953.

12. Ignat Ignatov, Oleg Mosin, Borislav Velikov, Enrico Baue, Influence of Isotopic Composition of Water with Varrying Deuterium Content In Composition With Mountain Water of Bulgaria on Human Longevity, Journal of Medicine, Physiology and Biophysics, 2014,vol.7:46-78.

13. Laszlo Kotai, Jozsef Lippart, Istvan Gacs, Bela Kazinczy, and Laszlo Vidra, Plant-Scale Method for the Preparation of Deuterium-Depleted Water, Industrial \& Engineering Chemistry Research 1999, vol. 3: 2425-2427.

14. Tatyana Strekalova, Matthew Evansa, Anton Chernopiatkod,, Yvonne Coucha, João Costa-Nunesb, Raymond Cespugliof, Lesley Chessong, Julie Vignisseh, Harry W. Steinbuschc, Daniel C. Anthonya, Igor Pomytkind,e, Klaus-Peter Lesch, Deuterium content of water increases depression susceptibility: The potential role of a serotoninrelated mechanism, Behavioural Brain Research 2015, vol 277: 237 244.

15. Hongqiang Wanga, Baohua Zhu, Zhiwei He, Hui Fu, Zhong Dai , Guoliang Huang, Binbin Li , Dongyun Qin, Xiaoyan Zhang, Lu Tian, Weiyi Fang, Huiling Yang, Deuterium-depleted water (DDW) inhibits the proliferation and migration of nasopharyngeal carcinoma cells in vitro, Biomedicine \& Pharmacotherapy 2013, vol. 67: 489496.

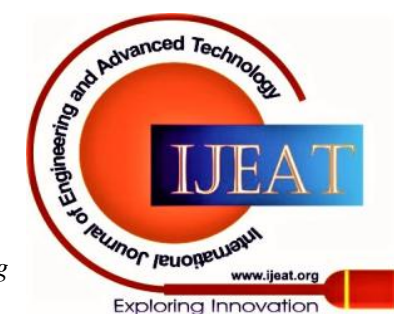

